\title{
Auditoria Administrativa aplicada a los procesos de adquisiciones de Liga Deportiva Cantonal Chimbo
}

\author{
Xavier Marcelo García García. \\ xgarcia@ueb.edu.ec \\ Universidad Estatal de Bolivar
}

\section{RESUMEN}

La presente investigación tiene como objetivo mostrar los resultados de la aplicación de una auditoria administrativa a Liga Deportiva Cantonal de San José de Chimbo, se buscó determinar el adecuado proceso de adquisiciones de bienes y servicios en esta institución. La importancia de la aplicación de una auditoria administrativa permite revisar los diferentes procesos administrativos y contables con la finalidad de encontrar errores en los mencionados procesos que permitan hacer las respetivas correcciones en beneficio de la institución

La investigación realizada fue de carácter documental se utilizó entrevistas y aplicación de cuestionarios al personal Directivo de la institución, así como la revisión de documentación física que reposa en sus archivos. Los resultados de la investigación indicaron que la empresa caso de estudio tuvo un nivel de confianza del $30 \%$ lo cual es medio bajo, generando un nivel de riesgo medio alto del 70\%. Las falencias detectadas fueron que no existen manuales de adquisiciones de bienes y servicios, no existen autorizaciones escritas por parte de la máxima autoridad para las adquisiciones, no existen registros contables de las adquisiciones, no existe una sola persona encargada de compras, no existe expedientes de las compras realizadas, no existen actas de custodio de bienes adquiridos, no cuenta con un archivo digital de los documentos existentes que corresponden a adquisiciones. Por lo que se recomienda la implementación de un manual de adquisiciones y un manual de control interno.

Palabras Clave: Auditoría administrativa, cuestionario, nivel de confianza, nivel de riesgo. 


\title{
Administrative Audit applied to the acquisitions processes of Liga Deportiva Cantonal Chimbo
}

\begin{abstract}
ABSTRAC
The present research aims to show the results of the application of an administrative audit a Liga Deportiva Cantonal de San Jose de Chimbo, I am looking for you to finish the proper process of purchasing goods and services at this institution.

The importance of the application of an administrative audit allows reviewing the different administrative and accounting processes in order to find errors in the aforementioned processes that allow making the respective corrections For the benefit of the institution.

The research carried out was of a documentary nature, interviews and questionnaires were applied to the directive staff of the institution as well as the review of physical documentation that rests in its files. The results of the investigation indicated that the case study company had a confidence level of $30 \%$ which is medium low generating a risk level médium high of $70 \%$. The shortcomings detected were that there are no manuals for the procurement of goods and services, there are no authorizations described by the highest authority for procurement, there are no accounting records of the acquisitions, there is no single person in charge of purchases, there are no records of the purchases made, no there are records of custodians of acquired goods does not have a digital archive of the existing documents corresponding to acquisitions. Therefore, the implementation of a procurement manual and an internal control manual is recommended.
\end{abstract}

Key Words: Administrative audit, questionnaire, confidence level, risk level.

Artículo recibido: 25 enero 2021 Aceptado para publicación: 28 febrero 2021

Correspondencia: xgarcia@ueb.edu.ec Conflictos de Interés: Ninguna que declarar 


\section{INTRODUCCIÓN}

La institución objeto de estudio es una sociedad no gubernamental sin fines de lucro que tiene como actividades principales la organización y gestión de competencias deportivas al aire libre o bajo techo con participación de deportistas profesionales o aficionados, por parte de organizaciones con instalaciones propias, se incluyen la gestión de esas instalaciones y la dotación del personal necesario para su funcionamiento, fue creada el 10 de marzo de 1951 se encuentra ubicada en el Cantón San José de Chimbo de la Provincia de Bolívar. Toda institución sea pública o privada necesita de un adecuado procedimiento de adquisiciones con la finalidad precautelar el uso adecuado de sus recursos económicos y materiales.

La auditoría es el acto de hacer una revisión profunda del proceso administrativo de la empresa, con el fin de corregir o reforzar sus operaciones para que esta logre una ventaja competitiva. (Chavarria, 2014)

La auditoría administrativa puede abarcar desde un área o departamento hasta la totalidad de la empresa, en el que se tendrá que descubrir y analizar todo su funcionamiento administrativo. Además incluye aspectos de su estructura organizacional, niveles, jerárquicos, relaciones interpersonales, operaciones, estándares de desempeño, desarrollo tecnológico entre otros. La auditoría administrativa es aplicable a cualquier tipo de organización ya que, sin importar el estilo de administración, los procesos o lineamientos que se sigan son sujetos a revisión. (Chavarria, 2014)

La auditoría administrativa es una herramienta que nos permite descubrir las deficiencias y áreas de oportunidad en una organización, ya que muchas veces el trabajo diario no nos permite ver que existen aspectos sujetos a mejora.

Es importante realizar una auditoria administrativa, al menos una vez al año, para descubrir cuáles son las fallas, en que se puede mejorar y que se está haciendo bien para fortalecerlo. En todas las organizaciones, sin importar su condición de privada, pública o social, se buscan mejoras continuas; así como procesos que les permitan sortear mejor los riesgos. La auditoría, al brindar información sobre como son, como funcionan y con que cuentan las organizaciones, es confiable, veraz y oportuna. (Chavarria, 2014)

El auditor aplicará procedimientos de evaluación del riesgo con el fin de disponer de una base para identificar y valorar los riesgos de error material en los estados financieros y en las afirmaciones. No obstante, los procedimientos de evaluación del riesgo por sí solos 
no proporcionan evidencia de auditoría suficiente y adecuada en la cual basar la opinión de auditoría. Los procedimientos de evaluación del riesgo incluirán, además de los señalados en esta guía, los siguientes:

a) Indagaciones ante la administración y ante otras personas de la entidad.

b) Procedimientos analíticos.

c) Observación e inspección.

El auditor obtendrá conocimiento de los siguientes elementos a fin de que pueda obtener un conocimiento suficiente y adecuado de la entidad que le permita llevar a cabo una adecuada evaluación de riesgos y sustente la estrategia inicial de auditoría:

a) Factores relevantes sectoriales y normativos, así como otros factores externos, incluido el marco de información financiera aplicable.

b) La naturaleza de la entidad, en particular:

- sus operaciones;

- sus estructuras de gobierno y propiedad;

- los tipos de inversiones que la entidad realiza o tiene previsto realizar; y

- el modo en que la entidad se estructura y la forma en que se financia para permitir al auditor comprender los tipos de transacciones, saldos contables e información a revelar que se espera encontrar en los estados financieros.

c) La selección y aplicación de políticas contables por la entidad, incluidos los motivos de cambios en ellas. El auditor evaluará si las políticas contables de la entidad son adecuadas a sus actividades y congruentes con el marco de información financiera aplicable, así como con las políticas contables utilizadas en el sector correspondiente.

d) Los objetivos y las estrategias de la entidad, así como los riesgos de negocio relacionados, que puedan dar lugar a errores materiales.

e) La medida y revisión de la evolución financiera de la entidad. El auditor debe aplicar su juicio profesional para determinar el grado de conocimiento necesario. (Aseguramiento, 2018)

Una actividad clave en la administración eficaz es procurar la adecuada protección de los activos, que constituye una de las actividades primordiales al establecerse los controles internos, para lo cual se requiere definir e implementar la adecuada segregación de funciones (...) (Pereira, 2019) 
El auditor deberá planear la naturaleza, oportunidad y alcance de los procedimientos que se utilizarán en determinadas cuentas (cuentas colectivas), así se conocerá las principales transacciones, además se identificaran áreas que puedan plantear riesgos más altos. Una adecuada planificación permite identificar hechos como transacciones y eventos poco comunes, como también los montos, las estimaciones y tendencias que puedan identificar declaraciones financieras que puedan requerir ramificaciones en el plan de auditoría. (Espino, 2015)

Toda entidad enfrenta una variedad de riesgos provenientes tanto de fuentes externas como internas que deben ser evaluados por la sus directivos quienes establecen objetivos generales y específicos, se identifican y analiza los riesgos de que dichos objetivos no se logren o afecten su capacidad para: Salvaguardar los bienes y recursos, construir y conservar su imagen, incrementar y mantener su solidez financiera (Garcia, 2021)

\section{METODOLOGÍA}

La presente investigación aplico el método deductivo lo que permite determinar recomendaciones específicas. Las técnicas utilizadas fueron:

- Inspección y revisión documental.

- Entrevistas.

- Aplicación de cuestionario.

\section{EJECUCIÓN DE LA AUDITORÍA ADMINISTRATIVA}

La auditoría se realizó a los procesos de adquisiciones de Liga Deportiva Cantonal de Chimbo con el objetivo de identificar y verificar el cumplimiento de normas y procedimientos obligatorios de la institución. El objetivo del presente trabajo fue auditar los procesos de adquisición realizados desde el 01 de enero al 31 de diciembre del 2019 de ser el caso identificar posibles falencias o irregularidades que se hayan dado durante el periodo antes mencionado.

Para el desarrollo de la auditoria, la planificación se distribuyó de la siguiente manera:

Tabla I: Planificación de Auditoría

\begin{tabular}{|l|l|c|c|}
\hline \multicolumn{1}{|c|}{ Etapa } & \multicolumn{1}{|c|}{ Actividad } & Tiempo & Responsable \\
\hline $\begin{array}{l}\text { Planificación } \\
\text { preliminar }\end{array}$ & $\begin{array}{l}\text { Visita y conocimiento } \\
\text { general de la Institución } \\
\text { mediante una entrevista } \\
\text { con su principal directivo. }\end{array}$ & 12 HORAS & X.G \\
\hline
\end{tabular}




\begin{tabular}{|l|l|c|c|}
\hline $\begin{array}{l}\text { Planificación } \\
\text { especifica }\end{array}$ & $\begin{array}{l}\text { Elaborar el cuestionario de } \\
\text { control interno para su } \\
\text { principal directivo. }\end{array}$ & 20 HORAS & X.G \\
\hline Ejecución & $\begin{array}{l}\text { Aplicación de técnicas y } \\
\text { procedimientos de } \\
\text { auditoria. }\end{array}$ & 16 HORAS \\
\hline $\begin{array}{l}\text { Comunicación } \\
\text { de resultados }\end{array}$ & $\begin{array}{l}\text { Elaboración del informe de } \\
\text { auditoría administrativa a } \\
\text { las adquisiciones de } \\
\text { LDCCH. }\end{array}$ & 12 HORAS & X.G \\
\hline
\end{tabular}

En la fase de planificación preliminar a través de una visita a sus instalaciones se pudo obtener un conocimiento previo del funcionamiento de la institución.

Luego de la planificación preliminar se desarrolló la planificación específica en el que se pudo realizar un análisis profundo de los procesos de adquisiciones y registros contables de los mismos, a continuación se continuó con la ejecución de la auditoria a través de las diferentes técnicas como la entrevista y observación de evidencias en las instalaciones de la Institución, en esta etapa se procedió a la aplicación de un cuestionario de control interno para determinar el nivel de riesgo y confianza, así como los hallazgos derivados de su aplicación.

\section{Tabla II: Cuestionario de Control Interno}

\begin{tabular}{|c|c|c|c|c|}
\hline $\mathbf{N}^{\circ}$ & Pregunta & Si & No & Observación \\
\hline 1 & $\begin{array}{l}\text { ¿La institución cuenta con un plan de } \\
\text { adquisiciones en el año? }\end{array}$ & $\mathrm{x}$ & & $\begin{array}{l}\text { Se lo realiza mediante el Plan } \\
\text { operativo anual. }\end{array}$ \\
\hline 2 & $\begin{array}{l}\text { ¿Existe un seguimiento a la ejecución } \\
\text { del presupuesto asignado para cada } \\
\text { año? }\end{array}$ & $\mathrm{x}$ & & $\begin{array}{l}\text { Mediante reportes que se envía } \\
\text { a la Coordinación Zonal de la } \\
\text { Secretaria del Deporte }\end{array}$ \\
\hline 3 & ¿Existe un manual de adquisiciones? & & $\mathrm{x}$ & $\begin{array}{l}\text { No existe en los archivos de la } \\
\text { Institución un manual de } \\
\text { adquisiciones. }\end{array}$ \\
\hline 4 & $\begin{array}{l}\text { ¿Todas las adquisiciones se las realiza } \\
\text { con la autorización y sumilla del } \\
\text { Presidente? }\end{array}$ & & $\mathbf{x}$ & \begin{tabular}{lr} 
Solamente & \multicolumn{2}{c}{ mediante } \\
comunicación verbal & o \\
posterior a la compra & se \\
comunica la adquisición. &
\end{tabular} \\
\hline 5 & $\begin{array}{l}\text { ¿Se realiza oportunamente el registro } \\
\text { contable de las adquisiciones? }\end{array}$ & & $\mathbf{x}$ & $\begin{array}{l}\text { No existen registros contables } \\
\text { de las adquisiciones, solamente } \\
\text { se pudo encontrar apuntes en un } \\
\text { cuaderno. }\end{array}$ \\
\hline
\end{tabular}




\begin{tabular}{|l|l|l|l|l|}
\hline $\mathbf{6}$ & $\begin{array}{l}\text { ¿Existe personal que este } \\
\text { específicamente encargado de las } \\
\text { adquisiciones? }\end{array}$ & & $\mathbf{x}$ & $\begin{array}{l}\text { Las compras las realiza } \\
\text { diferentes personas de la } \\
\text { institución. }\end{array}$ \\
\hline $\mathbf{7}$ & $\begin{array}{l}\text { iLas adquisiciones cuentan con un } \\
\text { expediente que justifiquen su } \\
\text { realización? }\end{array}$ & & $\mathbf{x}$ & $\begin{array}{l}\text { Solamente se pudo evidenciar } \\
\text { la existencia de facturas. }\end{array}$ \\
\hline $\mathbf{8}$ & $\begin{array}{l}\text { ¿Existen custodios responsables de } \\
\text { los bienes adquiridos por la } \\
\text { institución? }\end{array}$ & & $\mathbf{x}$ & $\begin{array}{l}\text { No existen actas de custodio de } \\
\text { bienes. }\end{array}$ \\
\hline $\mathbf{9}$ & $\begin{array}{l}\text { ¿Cuentacon un archivo físico y digital } \\
\text { de la documentación correspondiente } \\
\text { a adquisiciones? }\end{array}$ & $\mathbf{x}$ & $\begin{array}{l}\text { Solamente se cuenta con un } \\
\text { archivo físico en las } \\
\text { instalaciones de la institución. }\end{array}$ \\
\hline $\mathbf{1 0}$ & $\begin{array}{l}\text { ¿Los fondos económicos destinados a } \\
\text { las adquisiciones se encuentran } \\
\text { depositados en Instituciones } \\
\text { financieras? }\end{array}$ & $\mathbf{x}$ & $\begin{array}{l}\text { Al momento de realizar los } \\
\text { pagos se lo hace mediante } \\
\text { cheque o a través de retiros de } \\
\text { efectivo para cancelación con } \\
\text { fondos de autogestión. }\end{array}$ \\
\hline \multicolumn{1}{|c|}{ TOTAL }
\end{tabular}

\section{RESULTADOS}

Se realizó la ejecución de acuerdo a lo programado en donde se determinó el siguiente nivel de riesgo y de confianza de acuerdo a la siguiente tabla:

Tabla III: Matriz De Riesgo

\begin{tabular}{|c|c|c|}
\hline Rango & Riesgo & Confianza \\
\hline $0 \%-25 \%$ & BAJO & BAJO \\
\hline \multirow[t]{2}{*}{$26 \%-50 \%$} & MEDIO & MEDIO \\
\hline & BAJO & BAJO \\
\hline \multirow[t]{2}{*}{$51 \%-75 \%$} & MEDIO & MEDIO \\
\hline & ALTO & ALTO \\
\hline $76 \%-100 \%$ & ALTO & ALTO \\
\hline
\end{tabular}

La institución presenta el siguiente nivel de riesgo y de confianza, de acuerdo al cuestionario de control interno aplicado del $100 \%$ de respuestas el $30 \%$ son positivas lo que determina el nivel de confianza y el $70 \%$ son negativas lo que determina el nivel de riesgo, quedando ubicado en los rangos señalados a continuación: 
Tabla IV: Resultados de Aplicación de Matriz de Riesgo

\begin{tabular}{ccc}
\hline \multicolumn{1}{c}{ Rango } & Riesgo & Confianza \\
\hline $\mathbf{0 \%}-\mathbf{2 5 \%}$ & BAJO & BAJO \\
\hline $\mathbf{2 6 \%}-\mathbf{5 0 \%}$ & MEDIO & MEDIO \\
& BAJO & BAJO \\
\hline $\mathbf{5 1 \%}-\mathbf{7 5 \%}$ & MEDIO & MEDIO \\
& ALTO & ALTO \\
\hline $\mathbf{7 6 \%}-\mathbf{1 0 0 \%}$ & ALTO & ALTO \\
\hline
\end{tabular}

En las adquisiciones evaluadas, tenemos un nivel de confianza de medio bajo y un nivel de riesgo medio alto.

Los hallazgos en el proceso de adquisiciones fueron los siguientes: no existe, manual de adquisiciones, no existen autorizaciones escritas por parte de la máxima autoridad para las adquisiciones solamente mediante comunicación verbal o posterior a la compra se comunica la adquisición, no existen registros contables de las adquisiciones, no existe una sola persona encargada de compras, no existe expedientes de las compras realizadas, no existen actas de custodio de bienes adquiridos, no cuenta con un archivo digital de los documentos existentes que corresponden a adquisiciones.

\section{CONCLUSIONES}

- Luego de realizar la auditoria administrativa se logró cumplir con el objetivo que fue mostrar los resultados de la aplicación de una auditoria administrativa a Liga Deportiva Cantonal de San José de Chimbo y determinar el adecuado proceso de adquisiciones de bienes y servicios en esta institución.

- Liga Deportiva Cantonal Chimbo presento debilidades en su proceso de adquisiciones de bienes y servicios, por lo que se brindó la asesoría pertinente con la finalidad de mejorar estos procesos.

- La falta de políticas de procedimiento para adquisiciones así como la ausencia de documentación que la respalde, incrementa su riesgo administrativo y disminuye la confianza de los procesos realizados.

\section{RECOMENDACIONES}

- La presente investigación es una recopilación práctica y teórica de auditoria administrativa, que deberá estar disponible para el público en general en caso de que 
desee ampliar sus conocimientos acerca de la aplicación de una auditoría administrativa.

- Implementar un manual de adquisiciones que permitan un adecuado manejo de sus recursos económicos en los procesos de adquisiciones de bienes y servicios.

- Implementar un sistema de control interno que permita verificar el cumplimiento de las normas políticas y procedimientos referentes a los procesos de adquisiciones de bienes y servicios.

\section{REFERENCIAS BIBLIOGRAFICAS}

Aseguramiento, C. d. (2018). Guías de Auditoría. Mexico: Instituto Mexicano de Contadores Públicos .

Chavarria, C. (2014). Auditoría administrativa. Mexico: Editorial Digital UNID.

Espino, M. (2015). Fundamentos de auditoría. Mexico: Grupo Editorial Patria.

Garcia, X. (10 de Enero de 2021). dspace.espoch.edu.ec. Obtenido de dspace.espoch.edu.ec:

http://dspace.espoch.edu.ec/bitstream/123456789/5502/1/82T00408.pdf

Pereira, C. (2019). Control Interno en las empresas. Mexico: Instituto Mexicano de Contadores Públicos. 